\title{
Molecular dynamics study on the relaxation properties of bilayered graphene with defects
}

\author{
WEI ZHANG, JIU-REN YIN, PING ZHANG, YAN-HUAI DING** and YONG JIANG \\ Institute of Rheology Mechanics, Xiangtan University, Hunan 411105, China \\ *Author for correspondence (yhding@xtu.edu.cn)
}

MS received 5 August 2016; accepted 6 February 2017; published online 31 August 2017

\begin{abstract}
The influence of defects on the relaxation properties of bilayered graphene (BLG) has been studied by molecular dynamics simulation in nanometre sizes. Type and position of defects were taken into account in the calculated model. The results show that great changes begin to occur in the morphology after introducing defects into BLG sheets. Compared with point defects, line defects have a significant effect on the relaxation properties of BLG.
\end{abstract}

Keywords. Bilayered graphene; molecular dynamics simulation; defects; relaxation.

\section{Introduction}

Graphene, a well-known two-dimensional material, was first prepared in 2004 [1,2]. It has attracted considerable interest over the last few years on account of its extraordinary electrical, thermal and mechanical properties [3]. However, the vast majority of graphene sheets prepared by the chemical methods are double layer or even multilayers. A large number of researchers have studied the electronic structure and application of bilayered graphene (BLG). It was reported that the band gap of the BLG could be modified by applying an electric field $[4,5]$. Furthermore, the noise in BLG channels is lower than in single-layered graphene [6].

Relaxation performance is one of the most important aspects in the study of two-dimensional materials. Meyer et al [7,8] and Ishigami et al [9] found that suspended graphene was actually not flat and exhibited pronounced corrugations in nanoscale. A variety of defects, including topological defects [10,11], vacancy [12-17] and adatom [18-21], are inevitably produced in graphene during the preparation process. Yin et al [22] and Wu et al [23] have studied the influence of defects on relaxation properties of single-layer graphene. However, to our knowledge there are no studies on the relaxation properties of BLG yet. In this work, we used the molecular dynamics (MD) method to simulate the homeostasis evolution of single-layer graphene and BLG with point defects, line defects and topological defects. The distribution and population of the defects were taken into account in the calculated model.

\section{Physical models and simulation methods}

The position and type of the defects in graphene ribbons are shown in figure 1 . The length of the $\mathrm{C}-\mathrm{C}$ bond is $1.42 \AA$.
Two single graphene layers can be stacked and form bilayered structures [24]. The distance between the layers in bilayer graphene is $3.4 \AA[25,26]$. The dimension of simulation model is $58.2 \times 28.3 \AA$. There are four types of stacking in $\mathrm{BLG}$, which are $\mathrm{AA}, \mathrm{AB}, \mathrm{SP}$ and $\mathrm{BA}$ stacking. It has been proved that $\mathrm{AB}$ is the same as $\mathrm{BA}$, having an energy of $2.1 \mathrm{meV}$ per atom, a factor of 10 lower than the energy of AA-stacked graphene [27]. The large-scale atomic/molecular massively parallel simulator (LAMMPS) is a versatile and open source code for performing MD simulations of coarsegrained models. We employed the adaptive intermolecular reactive empirical bond order potential (AIREBO) to model the inter-atomic forces present in the covalent binding of carbon in the graphene. The AIREBO potential consists of three terms:

$$
E=\frac{1}{2} \sum_{i} \sum_{j \neq i}\left[E_{i j}^{\mathrm{REBO}}+E_{i j}^{\mathrm{LJ}}+\sum_{k \neq i} \sum_{l \neq i, j, k} E_{k i j l}^{\mathrm{TORSION}}\right] .
$$

The model was placed in a larger box $(80 \times 50 \times 30 \AA)$ with a certain distance between the edge of the model and the wall of the box. No external loads and constraints were applied on the boundary of graphene. In order to avoid the complex impact caused by the atomic thermal activation, the simulation was carried out at $0.01 \mathrm{~K}$ temperature, which was realized using a Nose-Hoover thermostat. The simulation time step is controlled to be $1 \mathrm{fs}$.

\section{Results and analysis}

\subsection{Structural defects discussion}

It is well known that structural defects do exist in graphene and may dramatically alter its properties. In fact, defects can also 

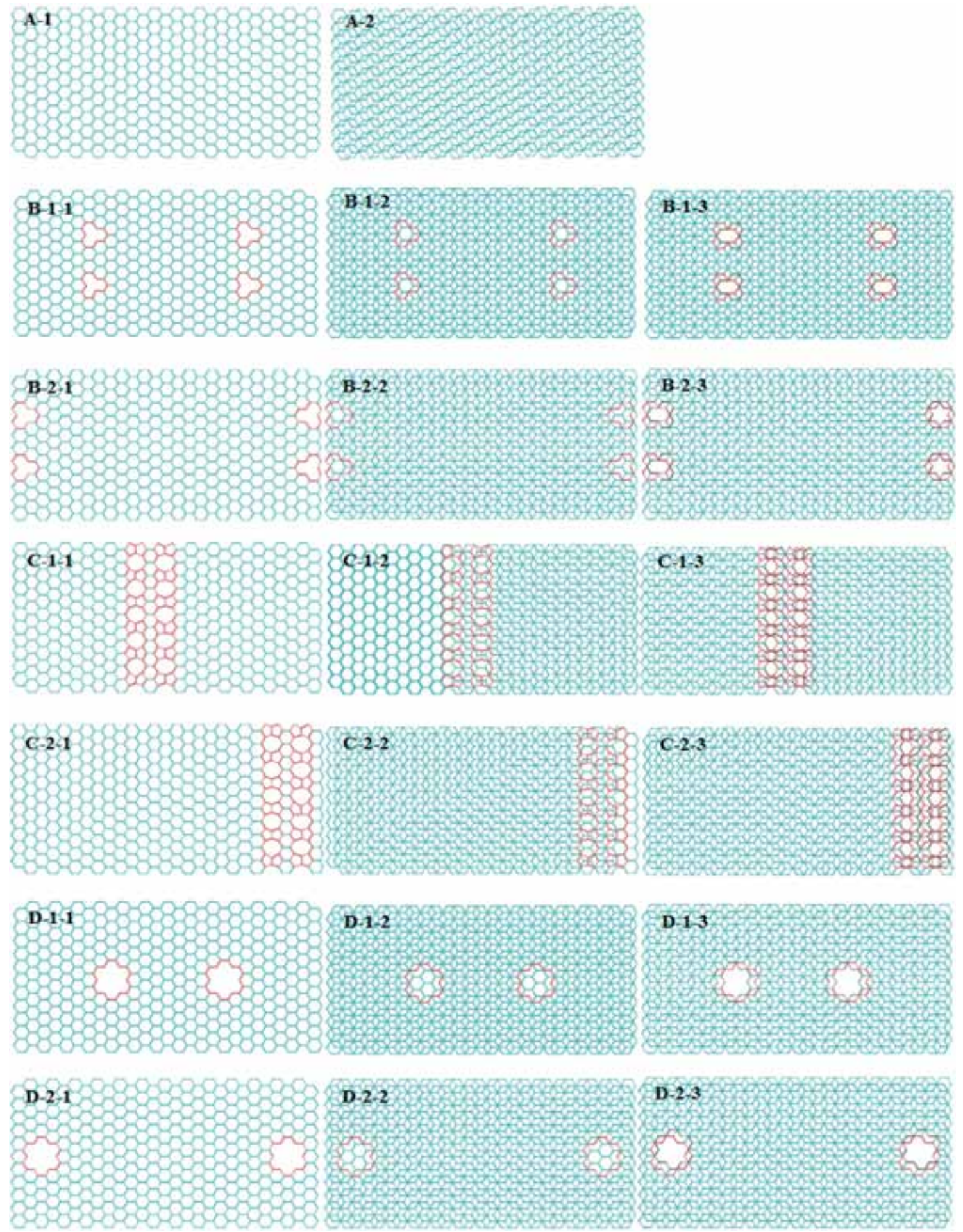

Figure 1. Schematic showing the type and positions of the defects: red lines represent defects in the top graphene layer; black lines represent defects in the bottom.

be deliberately introduced in graphene, for example, by irradiation or chemical treatments. For BLG, the interlayer distance is approximately $3.35 \AA$, as dictated by the weak van der Waals interaction between the layers. Therefore, defects can exist in both layers independently $[28,29]$. Structural defects may have some effects on the shape of stabilized graphene and different types would influence differently. The simplest defects in graphene are the Stone-Wales (SW) defects, which do not involve any removed or added atoms [11]. However, SW defects are unstable. Due to the high formation energy 


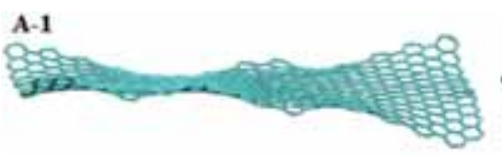

A-2

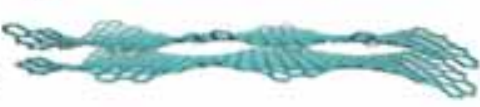

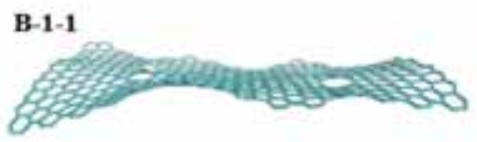

B-1-2

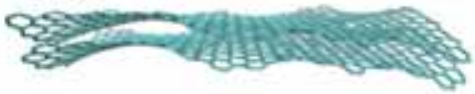

B-2-2

B-2-1

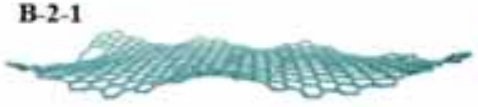

C-1-1
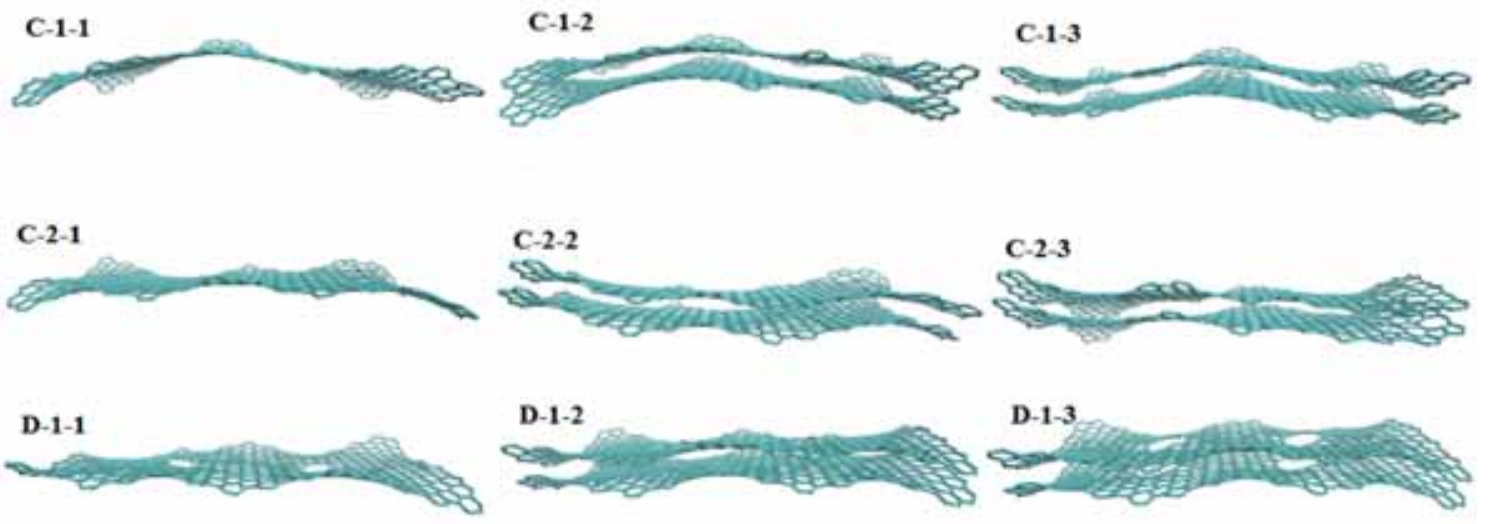

\section{D-1-2}
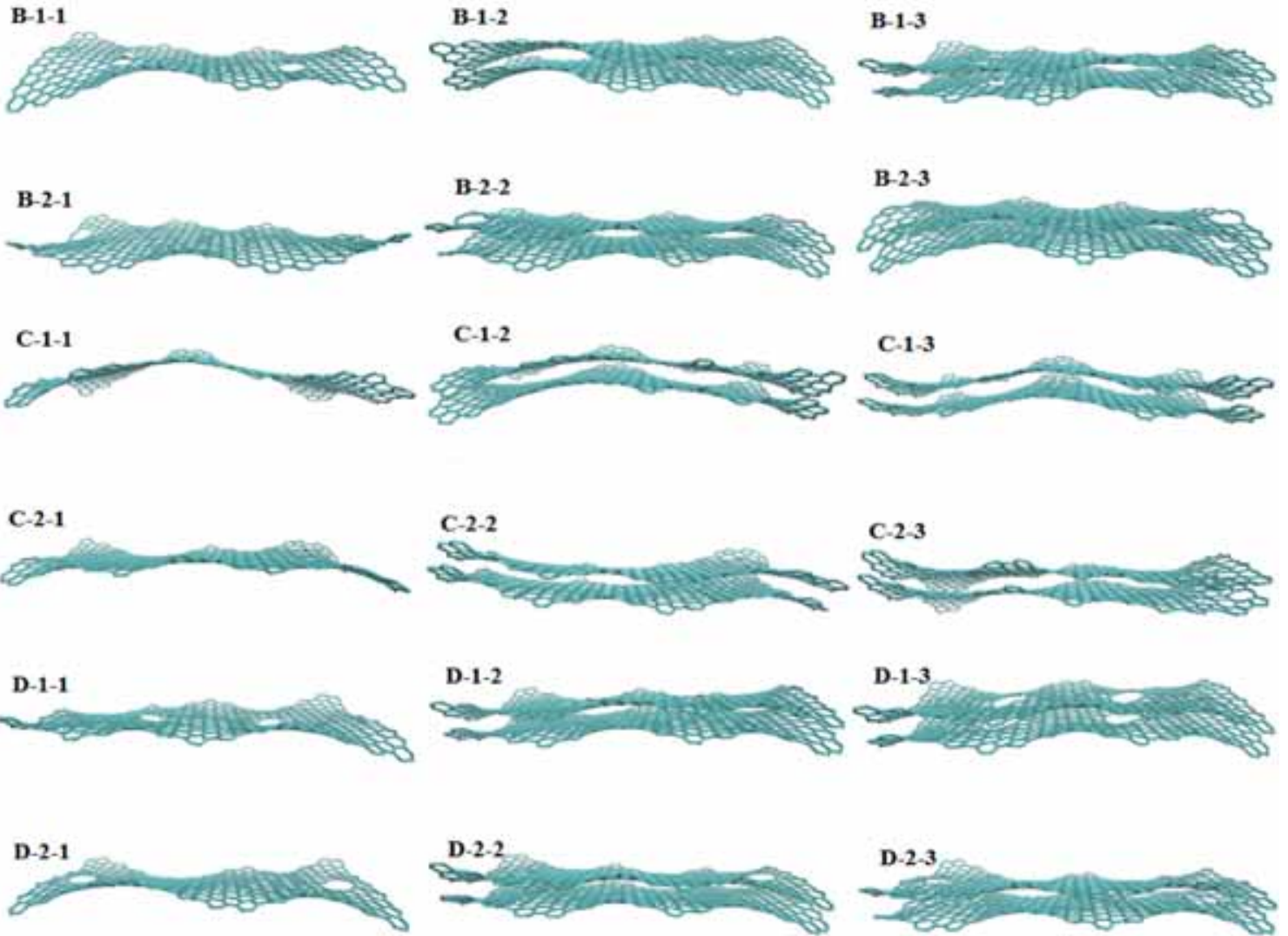

B-2-3
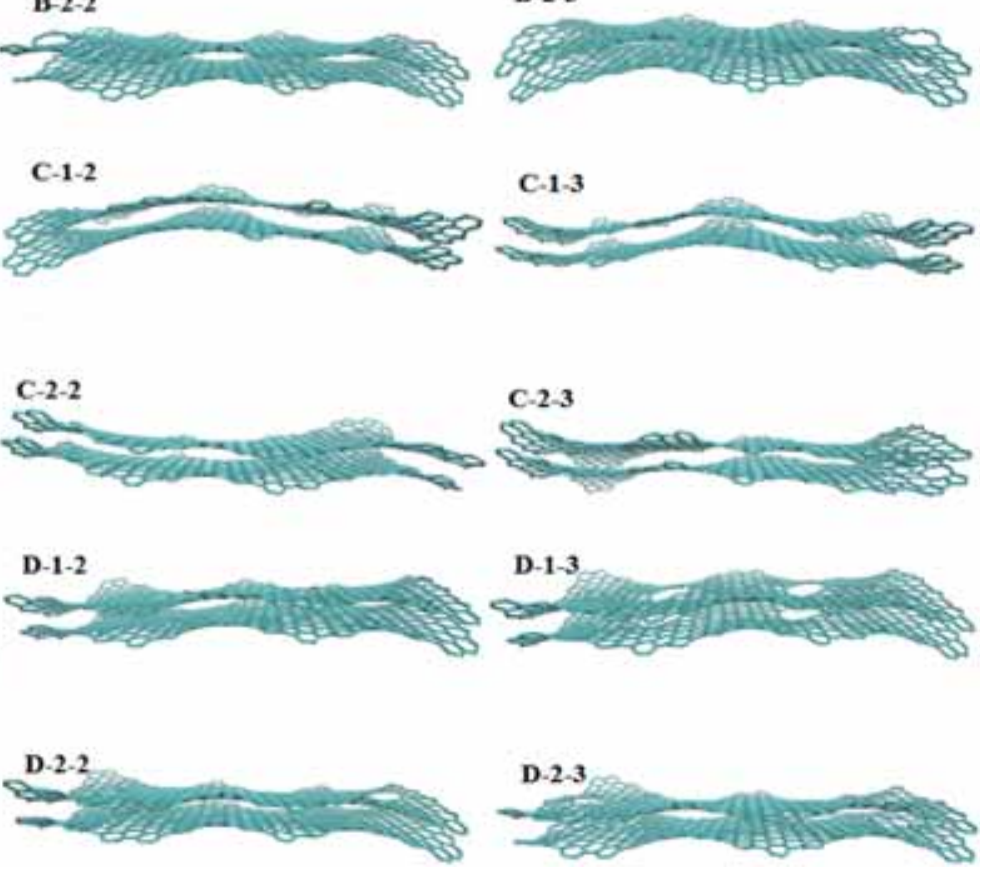

Figure 2. Atomic configuration of graphene after relaxation.

of SW defects, graphene would be reconstructed by rotating bonds and may relax to the unperturbed lattice [30]. In this paper, we investigated the stable shape of BLG influenced by different types of defects, including point defects, line defects and topological defects. We adopted single vacancies to represent point defects, because a missing lattice atom is the simplest defect in any material (as shown in model B). The line defect (expressed in model $\mathrm{C}$ ) is a special kind of grain boundaries, and it has been observed in experiments [31]. Line defects in model $\mathrm{C}$ were formed through the one-dimensional arrangement of some double vacancies, which could be created either by the coalescence of two single point defects or by removing two neighbouring atoms. Grain boundaries are usually formed by the 5-7 rings; with different arrangements, the grain boundary may remain flat or become inflected up to $72^{\circ}$. The topological defect in graphene (model D) is of various types, irregular shapes and different sizes. We employed the structure of graphene missing a hexagonal carbon ring as our topological defects calculation model. Before the relaxation, we calculated the chemical stability of topological defects by the DFT method.

\subsection{Relaxation}

Atomic configurations of graphene after complete relaxation are shown in figure 2. Single-layered graphene sheet is basically stable and the atoms have slight fluctuation, which is consistent with the relaxation results of graphene films reported in literature [32]. However, we found that the BLG starts rotating instead of fluctuation after the relaxation. The main reason may be the interaction between the two layers caused by the AB-stacked (Bernal) structure. The interaction between the upper and lower layers caused the model to generate the moment of inertia. In our calculation results, different types and positions of defects lead to different wavelengths and amplitudes of graphene.

The maximum kinetic energy and maximum normal displacement (z-axis) of carbon atoms were tracked and are presented in figure 3 . The calculation results have been classified into four groups.

(1) Graphene without defects

For the calculation of model A-1 (A-2), we found that, at the beginning of the relaxation, tiny corrugations appeared at 

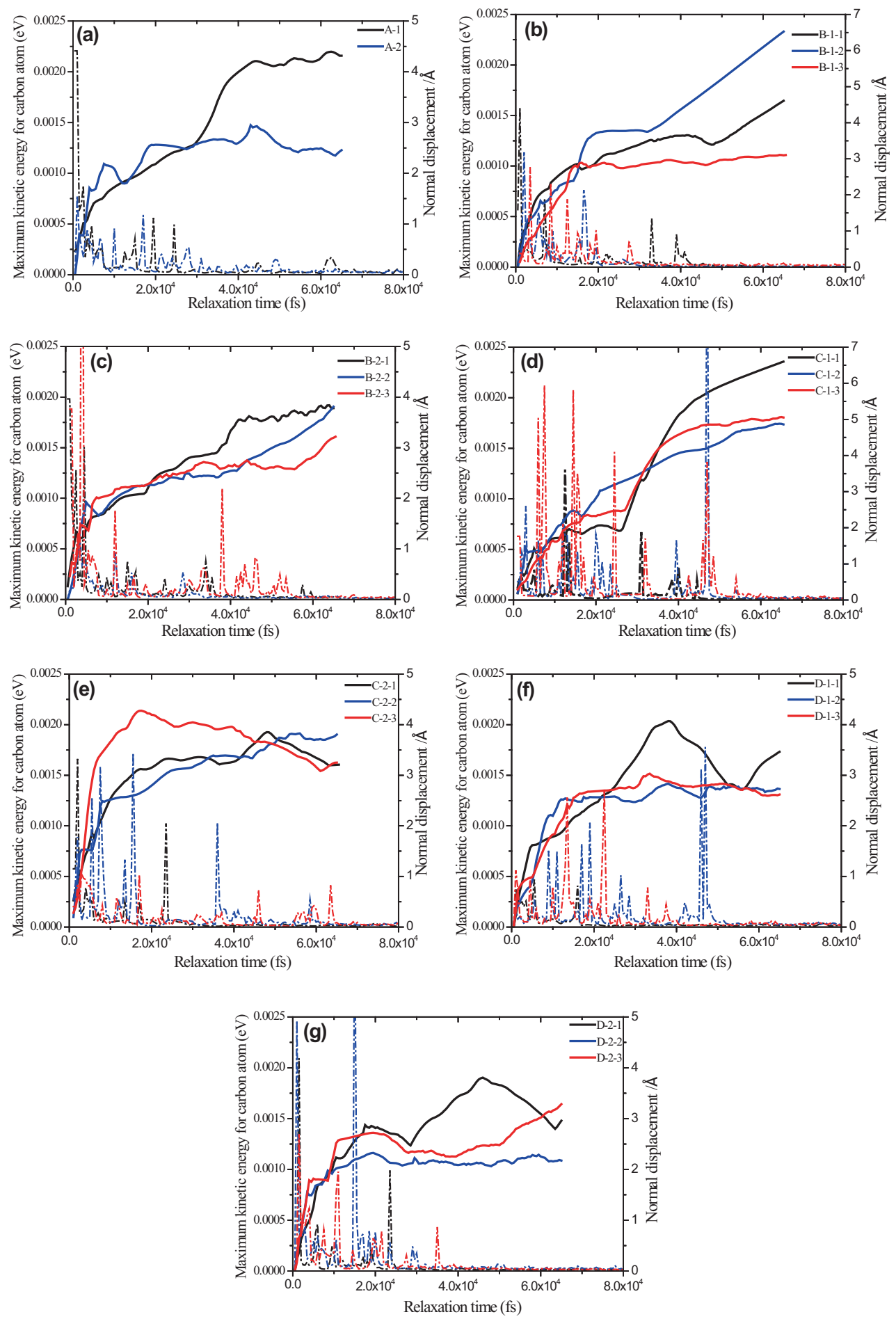

Figure 3. The maximum kinetic energy (dash-dot lines) and maximum normal displacement ( $z$-axis) (solid lines) of carbon atoms.

the edge of graphene films and increased with the relaxation time. After full relaxation, the film structure became stable and the maximum relaxation height of single-layer graphene was up to $4.4 \AA$ ( $3.0 \AA$ for BLG, see table 1). At the same time, we found that the corrugations existed only at the edge of films (both single-layer graphene and BLG) and the middle part of the film was flat. Ishigami et al [9] found that wrinkles appeared also in the middle part of the films. The main cause for this is that the models were calculated without applying any external loads and constraints. From an energy 
Table 1. Calculation results of models.

\begin{tabular}{|c|c|c|c|c|c|}
\hline & \multirow[b]{2}{*}{ Models } & \multicolumn{2}{|c|}{ Defects } & \multirow[b]{2}{*}{ Relaxation time (fs) } & \multirow{2}{*}{$\begin{array}{l}\text { Maximum normal } \\
\text { displacement }(\AA)\end{array}$} \\
\hline & & Type & Position & & \\
\hline A-1 & Single layer & - & - & $6.5 \times 10^{4}$ & 4.4 \\
\hline A-2 & Double layers & - & - & $5.0 \times 10^{4}$ & 3.0 \\
\hline B-1-1 & Single layer & \multirow[t]{6}{*}{ Point defects } & \multirow[t]{3}{*}{ Middle area } & $4.5 \times 10^{4}$ & 4.6 \\
\hline B-1-2 & Double layers & & & $3.0 \times 10^{4}$ & 3.8 \\
\hline B-1-3 & & & & $4.0 \times 10^{4}$ & 2.9 \\
\hline B-2-1 & Single layer & & \multirow[t]{3}{*}{ Boundary } & $4.0 \times 10^{4}$ & 3.6 \\
\hline B-2-2 & Double layers & & & $4.0 \times 10^{4}$ & 2.6 \\
\hline B-2-3 & & & & $5.5 \times 10^{4}$ & 2.7 \\
\hline C-1-1 & Single layer & \multirow[t]{6}{*}{ Line defects } & \multirow[t]{3}{*}{ Middle area } & $4.5 \times 10^{4}$ & 6.5 \\
\hline C-1-2 & Double layers & & & $6.0 \times 10^{4}$ & 4.9 \\
\hline C-1-3 & & & & $6.0 \times 10^{4}$ & 5.0 \\
\hline C-2-1 & Single layer & & \multirow[t]{3}{*}{ Boundary } & $3.0 \times 10^{4}$ & 3.8 \\
\hline C-2-2 & Double layers & & & $6.0 \times 10^{4}$ & 3.8 \\
\hline $\mathrm{C}-2-3$ & & & & $6.5 \times 10^{4}$ & 4.3 \\
\hline D-1-1 & Single layer & \multirow[t]{6}{*}{ Topological defects } & \multirow[t]{3}{*}{ Middle area } & $4.0 \times 10^{4}$ & 4.1 \\
\hline D-1-2 & Double layers & & & $5.0 \times 10^{4}$ & 2.8 \\
\hline D-1-3 & & & & $4.0 \times 10^{4}$ & 3.1 \\
\hline D-2-1 & Single layer & & \multirow[t]{3}{*}{ Boundary } & $5.0 \times 10^{4}$ & 3.8 \\
\hline D-2-2 & Double layers & & & $3.0 \times 10^{4}$ & 2.3 \\
\hline D-2-3 & & & & $4.0 \times 10^{4}$ & 2.7 \\
\hline
\end{tabular}

standpoint, the fluctuation curves indicate that they gradually tend to zero, which means the system is being relaxed to a stable state. Besides, the final maximum displacement was recorded when the maximum kinetic energy curves tended to zero. It should be noted that the maximum displacement of single layer is larger than that of BLG. However, the relaxation time of the single-layer graphene is longer than that of the BLG. We suggested that the van der Waals interaction between layers would limit the movement of atoms and make the film flatter.

(2) Graphene with point defects

Point defects in the middle area: As shown in figure 3b, the displacements of model B increase distinctly at the beginning of relaxation and achieve the balance after a certain time. Different relaxation times calculated from different type of defects were in the following order: B-1-1 > B-1-3 > B-1-2. BLG with perforative point defects (B-1-3) has the minimal relaxation shift. Type B-1-1 has a slight increase over that of type A-1 on the normal displacement, which is in agreement with the report [23]. The normal displace of B-1-2 is still increasing after $3 \times 10^{4} \mathrm{fs}$, and the growth trend is like an oblique line. The reason was BLG started rotating and affected the value of relaxation displacement. Therefore, the maximum relaxation displacement of model B-1-2 should be $3.8 \AA$. Based on energy, model B-1-1 achieved the stable state after $4.5 \times 10^{4} \mathrm{fs}$, whereas BLG models took more time for relaxation. Interestingly enough, the relaxation time of B-1-2 is the same as that of B-1-3. It implies that singlepoint defect has little influence on the relaxation of BLG.
Furthermore, compared with the single-layer point defects, it seems that the perforative point defects would reduce the normal displacement of BLG.

Point defects in the boundary: Compared with the middlearea point defects, the relaxation of point defects that lie in the boundary behaved differently both in normal displacement and in kinetic energy. Single-layer graphene with point defects on the edge has the maximal relaxation shift. BLG with perforative point defects on the edge has the longest relaxation time. From the kinetic energy, boundary point defects increased the relaxation time. With perforative point defects, the kinetic energy of model B-2-3 has a large fluctuation during relaxation. As shown in figure $3 \mathrm{c}$, the normal displacement of type B-2-1, B-2-2 and B-2-3 decreased slightly compared with perfect graphene. It indicates that the drift of the atoms is reduced when the point defects move to the edges.

(3) Line defects

Line defects in the middle: Single-layer graphene with line defects in the middle area has the largest displacement compared with other types of dfefects after relaxation. The kinetic energy of model C-1-1 changed with the normal displacement and reached zero state at $4.5 \times 10^{4} \mathrm{fs}$, which is the same for the perfect or point-defect graphene. However, the kinetic energy curves of BLG with line defects indicated a longer relaxation time. Moreover, during the relaxation, the kinetic energy of BLG with single-layer or perforative point defects fluctuated violently. As shown in figure $3 \mathrm{~d}$, the normal displacement of type C-1-1 is as high as $6.5 \AA$. For BLG (model C-1-2 and model C-1-3), the changes of maximum 
normal displacement presented the same increasing and had the same values. It indicated that line defects would superimpose fold deformation remarkably in single-layer graphene or BLG.

Line defects at the boundary: As shown in figure 3e, the kinetic energy of single-layer graphene decreased rapidly to zero at $3.0 \times 10^{4} \mathrm{fs}$. On the contrary, the relaxation time of model C-2-2 and C-2-3 increased to $6.0 \times 10^{4}$ fs and $6.5 \times 10^{4} \mathrm{fs}$, respectively. Compared with model A-1 and A-2, line defects will decrease the relaxation time of singlelayer graphene but increase the time of BLG. The reason we suggest is that it may be due to the structural asymmetry between layers and the number of atoms. The normal displacement of model C-2-1 is smaller than that of model C-1-1. The BLG with perforative line defects (C-2-3) has the largest normal displacement compared with that of model C2-1 and C-2-2. However, the maximum normal displacements of models C-2-1 and C-2-3 were smaller than that of models A-1 and A-2. We conclude that line defects that lie on the boundary may decrease the displacement of single-layer graphene or BLG. When perforative line defects appear at both the upper and lower layer, they may promote structural deformation.

(4) Topological defects

Topological defects in the middle area: Figure $3 \mathrm{f}$ describes the changes of displacement and kinetic energy during relaxation when topological defects are located in the middle area in graphene. The kinetic energy of model D-1-1 reaches the stable state at $4.0 \times 10^{4} \mathrm{fs}$, which is much shorter than that of model A-1. For BLG, the decreasing curves of kinetic energy are different. When topological defects appeared at only one layer, asymmetry between layers was remarkably increased. Therefore, model D-1-2 took more time for relaxation than did model D-1-3. D-1-2 has the minimum normal displacement and the longest relaxation time. The normal displacement of D-1-1 has a slight decrease compared with model A-2. The normal displacement of D-1-3 increased slightly compared with model A-2. However, the normal displacement of BLG with single-layer topological defects decreased slightly.

Topological defects at the boundary: As shown in figure 3g, the position of topological defects has a large effect on the changes of kinetic energy. Models D-2-1, D-2-2 and D-2-3 finally relaxed to the stable states at $5.0 \times 10^{4}, 3.0 \times 10^{4}$ and $4.0 \times 10^{4} \mathrm{fs}$, respectively. Compared with model D-1-1, the relaxation time of model D-2-1 increased. However, when defects lie in the boundary, the relaxation time of BLG is reduced. Model D-2-2 has the minimum normal displacement and the longest relaxation time. The normal displacement was reduced when topological defects were introduced into single-layer graphene and BLG sheets. Among them, the most obvious case is model D-2-2.

The relaxation time and maximum normal displacement are listed in table 1 . We find that the maximum normal displacement of the BLG with perforative defects is usually larger than that of the BLG with single-layer defects. This result implies that defects in the upper and lower layers, especially perforative defects, will interact and increase relaxation effects. As shown in table 1, the maximum normal displacement of BLG is smaller than that of single-layer graphene. It can be expected that corrugations will disappear when the number of layers is large enough. Among all the models, line defects located in the middle have the most significant effect on the relaxation properties of graphene.

\section{Conclusion}

The relaxation properties of single-layered graphene and BLG with three types of defects were studied by the MD method. BLG is not perfectly flat, with corrugated wrinkles appearing at the edge in a natural state as in single-layer graphene. The maximum normal displacement of BLG is smaller than that of single-layer graphene. Perforative defects are more likely to cause greater relaxation shift in BLG. Line defects have more significant effect on the relaxation properties of graphene compared with point and topological defects.

\section{Acknowledgements}

The financial support from the Natural Science Foundation of China (Numbers 21376199 and 51002128), Natural Science Foundation of Hunan Province (Number 2015JJ3115) and Scientific Research Foundation of Hunan Provincial Education Department (Numbers 17A205 and 15B235) is greatly acknowledged.

\section{References}

[1] Novoselov K S, Geim A K, Morozov S V et al 2004 Science 306666

[2] Novoselov K S, Jiang D, Schedin F et al 2005 Proc. Natl. Acad. Sci. USA 10210451

[3] Geim A K and Novoselov K S 2007 Nat. Mater. 6183

[4] Tsai S-J, Ho J-H, Chiu Y-H and Lin M-F 2010 Physica E: Low-Dim. Syst. Nanostruct. 422796

[5] Nanda B and Satpathy S 2009 Phys. Rev. B 80165430

[6] Lin Y-M and Avouris P 2008 Nano Lett. 82119

[7] Meyer J C, Geim AK, Katsnelson M, Novoselov K, Booth T and Roth S 2007 Nature 44660

[8] Meyer J, Geim A, Katsnelson M et al 2007 Solid State Commun. 143101

[9] Ishigami M, Chen J, Cullen W, Fuhrer M and Williams E 2007 Nano Lett. 71643

[10] Xu S, Irle S, Musaev D and Lin M 2007 J. Phys. Chem. C 111 1355

[11] Stone A J and Wales D J 1986 Chem. Phys. Lett. 128501

[12] Telling R H, Ewels C P, Ahlam A and Heggie M I 2003 Nat. Mater. 2333

[13] El-Barbary A, Telling R, Ewels C, Heggie M and Briddon P 2003 Phys. Rev. B 68144107 
[14] Krasheninnikov A, Nordlund K, Lehtinen P, Foster A, Ayuela A and Nieminen R 2004 Phys. Rev. B 69073402

[15] Krasheninnikov A, Nordlund K, Sirviö M, Salonen E and Keinonen J 2001 Phys. Rev. B 63245405

[16] Amorim R G, Fazzio A, Antonelli A, Novaes F D and da Silva A J 2007 Nano Lett. 72459

[17] Lee G-D, Wang C, Yoon E, Hwang N-M, Kim D-Y and Ho K 2005 Phys. Rev. Lett. 95205501

[18] Lehtinen P, Foster A, Ayuela A, Krasheninnikov A, Nordlund K and Nieminen R 2003 Phys. Rev. Lett. 91017202

[19] Nordlund K, Keinonen J and Mattila T 1996 Phys. Rev. Lett. 77699

[20] Talapatra S, Ganesan P, Kim T et al 2005 Phys. Rev. Lett. 95 097201

[21] Ma Y, Foster A, Krasheninnikov A and Nieminen R 2005 Phys. Rev. B 72205416

[22] Yin J-R, Wu W-H, Xie W, Ding Y-H and Zhang P 2015 Physica E: Low-Dim. Syst. Nanostruct. 68102
[23] Wu W, Yin J, Xie W et al 2015 IET Micro-Nano Lett. 10 693

[24] Bernal J 1924 Proc. R. Soc. London Ser. A 106749

[25] Brenner D W, Shenderova O A, Harrison J A, Stuart S J, Ni B and Sinnott S B 2002 J. Phys.: Condens. Matter 14783

[26] Lee C, Wei X, Kysar J W and Hone J 2008 Science 321385

[27] Popov A M, Lebedeva I V, Knizhnik A A, Lozovik Y E and Potapkin B V 2011 Phys. Rev. B: Condens. Matter 84 045404

[28] Ohta T, Bostwick A, Mcchesney J, Seyller T, Horn K and Rotenberg E 2006 Science $\mathbf{3 1 3} 951$

[29] Banhart F, Kotakoski J and Krasheninnikov A V 2011 ACS Nano 526

[30] Meyer J C, Kisielowski C, Erni R, Rossell M D, Crommie M F and Zettl A 2008 Nano Lett. 83582

[31] Lahiri J, Lin Y, Bozkurt P, Oleynik I I and Batzill M 2010 Nat. Nanotechnol. 5326

[32] Han T W and He P F 2010 Acta Phys. Sin. 593408 